\title{
Vaccine hesitancy communication: What counts as evidence
}

\author{
John Parrish-Sprowl \\ Indiana University Purdue University Indianapolis \\ Global Health Communication Center, Cavanaugh Hall 309 \\ 425 University Blvd \\ Indianapolis, IN 46202, United States
}

\begin{abstract}
The evidence base for vaccine hesitancy communication is generally confined to research focused on the content of the message rather than the process of communication. This has important consequences, because shifting focus to encompass the process of communicating, and not just on the content of content message being conveyed; opens the possibility of greater insight and understanding regarding the conversation health care workers have with those who are vaccine hesitant, creating increased chances of acceptance.
\end{abstract}

Keywords: Communication; Process; Evidence

\section{Introduction}

The increase in vaccine hesitancy over the past few years is a peculiar phenomenon. A growing number of people are delaying or putting off vaccination for themselves and/or their children due to concerns that are not founded on science or even general historical experience. While most people automatically accept the physician’s suggestion (or announcement) of vaccination, many

This is the author's manuscript of the article published in final edited form as:

Parrish-Sprowl, J. (2017). Vaccine hesitancy communication: What counts as evidence. Vaccine. https://doi.org/10.1016/j.vaccine.2017.09.064 
people in developed countries, including several with high levels of education, are not accepting vaccination despite the prevailing evidence of the benefits [1]. While the causes are varied and complex, one of the key issues concerns what counts as evidence of value and efficacy regarding vaccination for the person who expresses hesitancy. For some, the anecdote or the celebrity statement is of greater value than the research cited by their physician. We can disagree with a position predicated on such evidence, but what we cannot escape is that what counts as evidence is a choice we make in any situation, even as scientists or physicians, and it certainly impacts the choices made in vaccine hesitancy.

What counts as evidence in the support of a position or practice is a critical part of decision making. This is definitely the case in an era where the concept of "evidence based medicine" plays a defining role in determining acceptable, much less best practices, in medical care. Procedures, such as the Grading of Recommendations, Assessment, Development, and Evaluation (GRADE) method, have been developed to assess evidence in an effort to consider not only what counts as evidence but also what is the level of confidence one should place in the evidence at hand. Not surprisingly, those who have developed mechanisms such as GRADE consider the randomized controlled trial to be the gold standard for considering the quality of evidence in support of a conclusion regarding the principle, practice, or procedure being evaluated. This works well when the focus of evaluation is clear (e.g. the efficacy of a drug or vaccine). However, it becomes more problematic if the focus is something less clear, such as communication.

As Waisbord [2], Craig [3,4], and others have noted, the definition of communication is not a clear concept or activity, nor does everyone agree on a definition, theory, or meta theory of what it is and how it works. Depending on how one defines communication, the question of what 
counts as evidence can change dramatically. Many in health operate with the notion that communication is primarily a vehicle for the conveyance of information. The measure of communication quality is focused on clarity, completeness, and timeliness of information delivered. Thus the focus is almost solely on the content of the messages. Evidence for the effectiveness of communication is collected by both reach, recall, or perceived impact of a message that has been conveyed whether by media or in face-to-face situations such as a clinical setting. This data can tell us if people took in a message and can repeat it. However, in these circumstances, no matter what measures we choose, the focus is on the content of the communication and not the process itself.

Evidence of good process, however, is not the same thing as evidence of good content. Wellconceived messages, delivered poorly, may not have as much impact as poorly constructed messages delivered well. In other words, how we say something is often at least as important if not more important, than what we say. When we consider making evidence based decisions for patient provider interactions focused on vaccination, the process of the conversation should be our focus, not simply message content. More importantly, what we say occurs in the context of a conversation, not as a message standing alone, unfettered by the context of interaction between the parties. The process of conversation is where vaccine hesitancy will be addressed, not in a singular message that stunningly persuades a person to shift to acceptance.

However, quite often, studies that lead to the extant evidence base for vaccine hesitancy communication, are concerned with what is said and substantially ignores the way in which it is said or the context of the conversation in which it occurs [5]. This is not without value, but misses much of what is important when circumstances become more challenging, such as in the face of hesitancy. Thus, while we find good evidence for the approach to patients that focuses on 
the announcement (and therefore is assumptive) that today vaccination will take place, we have little in the way of evidence that indicates how this (and the rest of the conversation) should be conducted, especially if the person is hesitant or resistant. For this we must turn to research focused on the communication process irrespective of the content of the messaging.

A large and growing number of theorists and researchers in communication, complexity, and neuroscience, along with others exploring a quantum basis for social science explanations of human action, are adding depth to our understanding of the process nature of communication and the value of focusing more on conversation than on the content of a specific message [6,7]. While people process information in a rational, analytical way, they also interact and make decisions based on how they feel, even if their response is not consistent with the content of the message. For too long we have theorized and researched concepts such as risk and trust, key aspects of vaccine hesitancy, as if they were reasoned action or part of a health belief system without considering that they may be a manifestation of an intuitive feeling, part of an implicit memory, or based on an alternative domain of evidence that they accept as superior to the judgment of their health care professional (HCP) or the body of science that they offer as support of the reason to vaccinate.

Although a number of studies address the complex issues associated with communication between people, most of this research has not specifically centered on vaccine hesitancy. As a consequence, even though such research could inform HCPs regarding the conversational process with vaccine hesitant patients, it is often not considered because it is not content specific to the issue at hand. When content is considered without regard to the process of communication then such a criterion is reasonable. However, when the other aspects of interaction are the focus of analysis, and not simply the message content, then the rationale for excluding other research is 
less viable. This is not to say that research focused on the conversational process of vaccine hesitant-HCP interactions should not be conducted, but rather that evidence derived from conversation based research, especially on how trust and risk evolve in the flow, might serve as well as message based studies as guidance for practice.

For example, research has been done to support the notion that people are not as receptive to information when they are in a reactive of defensive state rather than a receptive frame of mind $[8,9]$ When the amygdala is activated, which can happen when people feel they are in a potentially adversarial conversation, then information is processed differently than when this is not the case. Further, some research suggests ways of interacting with people that can increase receptivity, irrespective of the specific issue, potentially setting the stage for the amelioration of hesitancy. In turn, if the HCP escalates the reactivity of the patient, then the hesitancy could move to resistance, making the efforts to increase vaccine uptake that much more difficult. Although this research has not specifically been conducted on vaccine hesitancy communication, there is little reason to believe that it does not apply to such conversations.

Just as we would have those experiencing vaccine hesitancy look at the best available science to make their decisions, so should HCPs regarding the evidence of communication. Focusing on the content of the message as if information is the only dimension of communication diverts attention from research that may well serve HCPs better as they attempt to develop the wherewithal to address the vaccine hesitant person in their offices, clinics, and communities. Evidence for communication efficacy is not simply about the content of the message, nor is it only about information and how it is analyzed as a basis for making choices. Perhaps this is why meta-analysis of research using the Theory of Reasoned Action finds that it typically accounts for only $27 \%$ of the variance [10]. Attitudes and beliefs are important in communication as well 
as decision-making, but they are not the only aspects of this process. So are intuitive feelings, implicit as well as explicit memories, and emotions. As a process, communication is more than message content; it is a range of interchanges, such that each party influences the flow, shaping what the other says. If we only count as evidence, message content about vaccines, then we miss examining evidence that could prove vital in addressing the problem of vaccine hesitancy. The narrow definition of communication that has dominated research in health and medical decisionmaking has led to an under consideration of evidence that could make a major difference in the management of the vaccine hesitancy phenomenon. 


\section{References}

[1] http://www.who.int/bulletin/volumes/86/2/07-040089/en/ [retrieved September 17, 2017].

[2] Waisbord S. Three challenges for communication and global social change. Commun Theory 2015:144-65.

[3] Craig RT. Communication theory as a field. Commun Theory 1999;9 (2):119-61.

[4] Craig RT. The constitutive metamodel: a 16-year review. Commun Theory 2015;25:356-74.

[5] Leask J, Kinnersley P. Physician communication with vaccine-hesitant paretns: the strat, not the end, of the story. Pediatrics 2015. https://doi.org/10.1542/peds.2015-1382. 180-182.

[6] Cozolino L. The neuroscience of human relationships: attachment and the developing social brain. New York: W.W. Norton; 2014.

[7] Sturmberg JP, Martin CM. Complexity in health: an introduction. In: Strumberg, Martin, editors. Handbook of systems and complexity in health. New York: Springer; 2013. p. 1 -7.

[8] Porges SW. The polyvagal theory: New insights into adaptive reactions of the autonomic nervous system. Cleveland Clin J Med 2009;76(suppl. 2):s86-90.

[9] Porges SW. The Polyvagal perspective. Biol Psychol 2007;74(2):116-43.

[10] Head KJ, Noar SM. Facilitating progress in health behavior theory development and modification: the reasoned action approach as a case study. Health Psychol Rev 2013. https://doi.org/10.1080/17437199.2013.778165. 